


\section{REVISTA DE PSICOLOGÍA}

Vol. XXIII. Segundo semestre 2005 Nº 2

\section{CONTENIDO}

\section{ARTÍCULOS}

Denise Benatuil, Alejandro Castro Solano y Alejandro Torres. Inteligencia práctica: un instrumento para su evaluación.

Dora Herrera Paredes y Hugo Morales Córdova. Comportamiento antisocial durante la adolescencia: teoría, investigación y programas de prevención.

James S. Fleming y César Merino Soto. Medidas de simplicidad y de ajuste factorial: un enfoque para la evaluación de escalas construidas factorialmente.

Cecilia Chau y Stephan Van den Broucke. Consumo de alcohol y sus determinantes en estudiantes universitarios limeños: estudio de focus group.

Inés Kudó, Tesania Velásquez, Mónica Iza, Alicia Ángeles, César Pezo del Pino y Patricia Martínez U. Una experiencia de intervención en crisis en el sur del Perú: Brigadas Psicológicas. 


\title{
Consumo de alcohol y sus determinantes en estudiantes universitarios limeños: estudio de focus group
}

\author{
Cecilia Chau ${ }^{1}$ \\ Pontificia Universidad Católica del Perú \\ Stephan Van den Broucke ${ }^{2}$ \\ Universidad Católica de Lovaina
}

\begin{abstract}
Se presenta un estudio cualitativo -focus group- para conocer los hábitos de consumo del alcohol y los principales determinantes de su uso entre estudiantes universitarios de la ciudad de Lima. Se organizaron cuatro grupos homogéneos (por género y nivel socio económico) con 5 a 9 participantes. El análisis de contenido reveló que los estresores experimentados son similares a los reportados por los adolescentes occidentales, sin embargo, reportaron estrés adicional por las dificultades económicas y el control de peso (entre las mujeres). Las expectativas sobre el alcohol fueron positivas y reflejaron lo mismo que en los adolescentes norteamericanos (mejora social, reducción de la tensión y mejora de habilidades motoras/cognitivas), aunque también se mencionaron expectativas adicionales (placer, diversión, pérdida de inhibiciones, auto confianza y aceptación del grupo). Otras fueron consideradas menos importantes (mejora sexual, incremento de la activación y deterioro cognitivo/motor). La autoeficacia para rechazar el alcohol no fue percibida como un determinante importante entre los participantes.

Palabras clave: consumo de alcohol, estresores, autoeficacia, expectativas, estudiantes universitarios.
\end{abstract}

\begin{abstract}
Alcohol consumption and its determinants among Peruvian university students: A focus group study

A qualitative study was performed using the focus group methodology with first year university students in Greater Lima, to learn about their drinking habits and explore the main determinants of their alcohol consumption. Four homogeneous focus groups (by gender and social class) were organized, with group sizes between 5 and 9 . Content analysis of the discussions revealed that the stressors experienced by these students are similar to those of other western adolescents, yet that economic difficulties and weight concerns (among females) provide additional stress. Expectancies towards alcohol were predominantly positive and partly reflected those of American adolescents (social enhancement, tension reduction and improved cognitive/motor abilities), although additional expectancies were mentioned (pleasure, diversion, loss of inhibitions, selfconfidence and group acceptance) and others were considered less important (sexual enhancement, increased arousal, and cognitive/motor impairment). Self-efficacy to refuse alcohol was not perceived as an important determinant of alcohol use.
\end{abstract}

Key words: alcohol consumption, stressors, self-efficacy, expectancies, university students. 

El uso del alcohol y otras drogas psicoactivas es muy común entre los estudiantes universitarios en muchos países (Minugh \& Harlow, 1994; O' Conell \& Patterson, 1989; Organización Mundial de la Salud, 1994; Thorne \& De Blassie, 1985). En el Perú, la investigación epidemiológica ha mostrado que el $65 \%$ de estudiantes universitarios consume alcohol (CEDRO, 1993, 1997). Esto hace del alcohol la sustancia más utilizada entre los estudiantes, precediendo al tabaco, las medicinas prescritas y las drogas ilegales. A pesar de que la prevalencia del consumo problemático de alcohol entre la población universitaria aún no es bien conocida, se ha encontrado que este tipo de consumo es la forma de dependencia a sustancias más importante en la población peruana (Saavedra, 1997). Debido a que su consumo excesivo puede causar diversos problemas psicológicos y físicos, tales como dependencia, depresión, ansiedad, cirrosis, pancreatitis, úlceras y cáncer, así como grandes problemas sociales, se hace necesaria la prevención primaria del uso de alcohol.

Para desarrollar programas de prevención efectivos es necesario comprender los determinantes conductuales correspondientes. Según se desprende de la literatura, entre los principales determinantes del uso de sustancias en los jóvenes se encuentran las formas como ellos afrontan los estresores de la vida (Seiffge-Krenke, 1995; Wills \& Shiffman, 1985), las expectativas que tienen sobre

1 Doctora en Psicología. Profesora del Departamento de Psicología de la PUCP. Sus temas de interés son la psicología de la salud, adicciones y adolescencia. Correo electrónico: cchau@ pucp.edu.pe

2 Doctor en Psicología. Profesor de la Facultad de Psicología de la Universidad Católica de Lovaina. Correo electrónico: Stephan.VandenBroucke@psy.kuleuven.be 
los efectos de las sustancias (Brown, Goldman, Inn \& Anderson, 1980; Goldman, Brown \& Christiansen, 1987), la presión de los pares y las normas percibidas (Johnson, 1988; Petraitis, Flay \& Miller, 1995), así como la autoeficacia para rechazar las sustancias que les son ofrecidas (Oei, Fergusson \& Lee, 1998). Sin embargo, estos conceptos y los modelos explicativos sobre los determinantes implicados en el uso de sustancias han sido principalmente estudiados en América del Norte y Europa. Dado que las explicaciones causales sobre la salud y la enfermedad varían de una cultura a otra (Murdock, 1980; Schweder et al. como se cita en Marks, Murray, Evans \& Willig, 2000), podría ocurrir que el enfoque social cognitivo sobre el uso de sustancias no se aplique satisfactoriamente a la cultura latina. Incluso el contenido específico de algunos conceptos antes mencionados podría diferir, dependiendo de la cultura y el subgrupo de interés. Por ejemplo, es probable que los estresores de vida específicos experimentados por los estudiantes peruanos o sus expectativas sobre el alcohol difieran de aquellos reportados por sus pares americanos o europeos. Por lo tanto, antes de que estos conceptos puedan ser aplicados para explicar el consumo de alcohol entre los estudiantes peruanos, su relevancia debería ser verificada en la cultura peruana.

Con este propósito, se realizó un estudio cualitativo utilizando la metodología de grupo focal en un grupo de estudiantes universitarios de Lima. Los principales objetivos de este estudio fueron:

1. Explorar los principales problemas y estresores.

2. Conocer los hábitos de consumo de bebidas alcohólicas.

3. Identificar los factores asociados al consumo de alcohol (tales como presión de grupo, expectativas y autoeficacia). 


\section{Metodología}

\section{Participantes}

Los participantes de este estudio fueron universitarios hombres y mujeres, matriculados en el primer año de estudios, en universidades privadas de Lima. Ellos fueron seleccionados para la investigación utilizando un muestreo no probabilístico, el que incluyó los siguientes pasos. Primero, se contactaron posibles participantes entre los matriculados en universidades seleccionadas. Para garantizar la confidencialidad de la investigación y asegurar que los estudiantes pertenecieran a la muestra de interés, se les administró un cuestionario de filtraje para identificar los niveles socioeconómicos de los mismos. Sólo aquellos estudiantes que cumplían con las características requeridas para el estudio fueron invitados a participar en las sesiones de grupos focales. Las edades de los participantes estuvieron entre los 17 y los 20 años; además, ellos pertenecían a los niveles socioeconómicos medio y medio alto.

Para cumplir con los objetivos del estudio, se establecieron cuatro grupos focales: uno con estudiantes mujeres de nivel socioeconómico (NSE) alto, otro con estudiantes hombres de NSE medio, uno tercero con estudiantes mujeres de NSE medio alto y un último grupo con estudiantes hombres de NSE medio alto también. Para contar con un promedio de 8 participantes por grupo, de 5 a 11 estudiantes fueron invitados para cada sesión. El tamaño final de los grupos fue de 9 estudiantes mujeres de NSE medio, 8 estudiantes hombres de NSE medio, 5 estudiantes mujeres de NSE medio alto y 9 estudiantes hombres de NSE medio alto. 


\section{Procedimiento}

Las discusiones de los grupos focales, los cuales duraron casi dos horas cada uno, fueron realizadas por un facilitador entrenado. Se crearon las condiciones necesarias para la expresión libre y espontánea de los participantes favoreciendo de la espontaneidad, los estilos de interacción informales y la inclusión de dinámicas grupales. Al mismo tiempo, la discusión se centró en los temas de interés, con el fin de permitir conversaciones profundas. Con tal fin, se preparó antes de las reuniones una guía en la que se tomaron en consideración los objetivos de la investigación.

Para identificar los estresores vitales experimentados por los participantes, la discusión se centró en las dimensiones incluidas en el Cuestionario de Problemas (Seiffge-Krenke, 1995), tocándose los siguientes temas: 1) presión de pares, 2) vida social / contacto con el sexo opuesto, 3) familia, 4) estudios, 5) finanzas, 6) trabajo, 7) tensiones raciales, y 7) salud. Con respecto a los hábitos de consumo de alcohol, se tocaron los siguientes temas: 1) tipo de bebida alcohólica consumida, 2) razones para beber, 3) toma de decisión al comprar las bebidas alcohólicas, 4) facilidades para comprar bebidas alcohólicas, 5) situaciones de consumo de las bebidas alcohólicas, y 6) lugares usualmente concurridos para beber. En cuanto a la influencia de los pares, dos temas se incluyeron: 1) bebidas alcohólicas consumidas por el grupo de pares, y 2) la cantidad de presión ejercida por los pares para beber bebidas alcohólicas. Para focalizar en las expectativas relacionadas con los efectos del alcohol, se administraron tres escalas del Cuestionario de Expectativas del Alcohol versión Adolescentes (AEQ-A; Christiansen, Goldman \& Inn, 1982): 1) el alcohol puede promover o impedir la conducta social, 2) el alcohol mejora las habilidades cognitivas y motoras, 3) el alcohol relaja y reduce el estrés. Finalmente, la discusión respecto a la autoeficacia fue guiada por los ítems del Cuestionario de Autoeficacia frente a la 
Tentación de Fumar (Schwarzer, 1993), adaptada para el uso de alcohol.

En cada uno de los temas antes mencionados, el facilitador primero solicitó comentarios generales sobre el tópico de interés y luego se centró en los ítems específicos. Por ejemplo, cuando se discutieron los estresores vitales experimentados por los participantes, se hizo una pregunta general sobre cuáles se percibían como los más estresantes y subsecuentemente, los temas se constituyeron en las dimensiones del Cuestionario de Problemas. Este procedimiento se repitió para cada tópico. Para aclarar la discusión, se utilizaron materiales auxiliares tales como marcadores, lápices de colores, resaltadores, revistas, tijeras, goma, etc. Por ejemplo, las expectativas sobre el alcohol podían ser representadas por un collage, y las características significativas de la evaluación de la autoeficacia fueron anotadas en grandes hojas de papel.

Las cuatro sesiones fueron observadas por la investigadora principal y tres colaboradores, quienes se ubicaron detrás de un espejo unidireccional, habiéndose advertido a los participantes de la presencia de dichas personas con el fin de evitar obstrucciones a lo largo de las sesiones. Todos los observadores recibieron entrenamiento en técnicas de observación antes de que se realizaran las sesiones. Además, las discusiones de los grupos focales fueron grabadas en VHS y audio para facilitar el análisis posterior de los materiales. Finalmente, durante las sesiones el facilitador podía interactuar con el investigador principal con el fin de clarificar ciertos aspectos de la discusión.

\section{Análisis de datos}

Se hicieron las transcripciones de cada discusión a partir de las notas de los observadores y utilizando las grabaciones de video y 
audio como apoyo. Para analizar estas transcripciones, se realizó un análisis de contenido siguiendo los siguientes pasos:

1. Primero, cada discusión fue dividida en fragmentos de textos relacionados con los temas principales y establecidos en la guía.

2. En cada fragmento, cada uno de los observadores por separado, resaltó las palabras clave que representaran las ideas principales o los grupos lógicos de información.

3. Luego, las palabras clave fueron agrupadas en categorías por cada uno de los observadores, también por separado.

4. Las categorías fueron comparadas entre los observadores para identificar similitudes. Si había suficiente acuerdo, dichas categorías y su significado (inferido de los fragmentos del texto) fueron replanteados en afirmaciones. Si no había acuerdo suficiente, se veía un consenso entre los observadores sobre el significado exacto de los temas logrados durante la sesión.

5. Finalmente, se realizó un análisis comparativo de los datos según género y clase social de los participantes.

\section{Resultados}

\section{Estresores vitales}

Cuando se les preguntó sobre las fuentes de estrés generales en su vida como estudiantes universitarios y sobre las formas cómo ellos afrontaban estas presiones, no se observaron diferencias entre los grupos según sexo y NSE. Tanto los chicos de NSE medio y alto, como las chicas de NSE medio alto, reportaron mayores niveles de estrés y más eventos estresantes en su vida diaria que las chicas de NSE medio. La principal fuente de estrés para los chicos de NSE medio fue la falta de dinero, mientras que para los chicos y chicas de NSE alto fue la responsabilidad de estar en 
la universidad y temas relacionados con la familia. Esto último también fue uno de los mayores factores de estrés para las chicas de NSE medio. Otros problemas mencionados espontáneamente, principalmente por los chicos, fueron las drogas, la necesidad de trabajar, estudiar, la orientación vocacional, la desorganización personal y la falta de relaciones interpersonales con chicas, así como el transporte público. Las chicas de NSE medio alto reportaron, generalmente, el control del peso y la necesidad de verse atractivas, las relaciones con los chicos, la falta de confianza entre amigos y la economía. Los chicos de NSE medio alto manifestaron que el dinero era su problema principal, en segundo lugar estaban los problemas familiares y en tercer lugar el consumo de drogas.

Cuando se aludió a los ítems mencionados en el Cuestionario de Problemas, destacaron los siguientes temas:

\section{Universidad y estudios}

La universidad y los estudios son experimentados como generadores de estrés debido a que elevan la responsabilidad y presionan por el logro de un buen desempeño en los cursos y altas calificaciones. Esta responsabilidad fuerza a los estudiantes a proyectarse en el futuro como "buenos profesionales" lo que, especialmente para las chicas de NSE medio alto, es un estresor. Los estudiantes también reaccionan negativamente frente a la competitividad que perciben entre los estudiantes de otras universidades. Esto es intensificado por la presión de obtener una alta calificación y la anticipación de la posibilidad de fracasar en la obtención de un trabajo en el área en la que estudiaron y se prepararon. Para lidiar con esta responsabilidad, los estilos de afrontamiento típicos fueron alejarse y concentrarse en sus estudios o, por lo contrario, relajarse. Salir y tomar unos tragos con los amigos 
como una forma de afrontamiento era reservado para ocasiones especiales, como por ejemplo cuando un trabajo era terminado o para celebrar el final de los exámenes parciales o finales.

Las chicas de NSE medio estuvieron más preocupadas por la falta de tiempo para cumplir con los cursos, la falta de materiales para trabajar y la relación de trabajo con los profesores, quienes a menudo fueron percibidos como "usualmente no disponibles". Los exámenes provocaban grandes niveles de estrés y los participantes reportaron que cuando éstos se aproximaban, a menudo ellos y sus compañeros se encontraban de mal humor. Los estudiantes de NSE medio también mencionaron la presión de hacer muchos trabajos al mismo tiempo y sentían que no tenían tiempo suficiente para tener todo listo, ya sea en la universidad, en la casa o el trabajo.

Los estudiantes hombres y mujeres de NSE medio también mencionaron la presión y la demanda de estudio por parte de sus padres. Los estudiantes hombres pensaban que la presión era mayor para ellos que para las estudiantes mujeres debido a que ellas "eventualmente serán mantenidas por sus esposos", mientras que ellos "tendrán que mantener a sus familias". Los estudiantes hombres de NSE medio alto también percibieron altos niveles de presión parental con relación a los estudios y al ser competitivo. Por otro lado, ellos también indicaron que las mujeres de hoy en día son fuertes competidoras también.

Otro estresor es la combinación de trabajo y estudio. Debido a la falta de apoyo para los estudiantes que combinan ambas actividades, los cursos son aprobados con bajas notas, lo cual es frustrante y desconsolador. Otros estudiantes prefirieron salir de la universidad y relajarse, entre otras cosas, tomando un trago. 
Relaciones con la familia

Las chicas que provinieron de NSE medio alto muestran una fuerte preocupación por los problemas que pudieran presentarse en sus hogares, básicamente por el poco tiempo que sienten que dedican a la familia dado que su mayor preocupación es la universidad. Por otro lado, los estudiantes hombres de NSE medio se mostraron preocupados por no ser capaces de ayudar a sus familias.

Las peleas con los hermanos también crean tensión, ya sea por las diferencias de edad o por los diferentes intereses y necesidades.

Con respecto a las relaciones con los padres, los estudiantes hombres del NSE medio alto reportaron tener discusiones por dinero, además de que también mencionaron la separación y el divorcio. En contraste, las estudiantes mujeres de NSE medio sentían más estrés por la falta de libertad y la sobreprotección de sus padres (e. g. "los padres te controlan mucho porque eres mujer"). Ellas mencionaron que sus padres sentían temor de los extraños o no confiaban en los amigos del sexo opuesto. Los estudiantes hombres del mismo NSE también reportaron la falta de libertad, pero sus conflictos con sus padres fueron más percibidos como la falta de comprensión de sus necesidades de diversión (e. g. "ellos no me dejan salir o cierran la puerta temprano, por lo que yo no puedo llegar después del toque de queda"). Para afrontar estos problemas familiares, la mayoría de participantes recurrieron a los amigos, pero también fue mencionado el consumo de drogas, ya sea para olvidar problemas y divertirse o para ser aceptado por el grupo. 
Relaciones con los pares

Aunque para la mayoría de participantes entrar a la universidad implicó que ellos tuvieron que encontrar su propio camino dentro de un grupo de personas con diferentes creencias, intereses y preferencias, ello no les impidió hacer nuevos amigos. A menudo, las amistades se establecen en diversos niveles y se tienen encuentros y desencuentros. Estas diferencias pueden causar problemas entre los amigos, por ejemplo, cuando uno se da cuenta que éstos presionan para ir a ciertos lugares o para hacer cosas que uno no desea. Al respecto, los participantes hombres específicamente mencionaron el préstamo de dinero o la falta de cooperación en los trabajos de grupo. Mientras que las participantes mujeres con frecuencia reportaron problemas relacionados con la envidia entre enamoradas, celos de los enamorados y la hipocresía (por ejemplo, cuando sus amigos murmuran sobre ellas). Usualmente estos problemas crean tensión y separación en los grupos o llevan a tomar partido por los amigos.

\section{Relaciones con el sexo opuesto}

Todos los participantes reportaron dificultades al tratar de establecer relaciones con alguien del sexo opuesto. La idea de aproximarse a alguien provoca cierta tensión, que con frecuencia lleva a no conversar con las personas que se encuentran atractivas. Una forma común para afrontar esta tensión es tomar un trago con los amigos para darse confianza y "tomar valor". Los problemas identificados con mayor frecuencia en las relaciones de pareja fueron la falta de confianza y de comunicación. Una razón importante para pelear fue la deshonestidad y el relacionarse con una tercera persona. A menudo, este tipo de problemas resultó en la ruptura de la pareja y con la persona "traicionada" sintiéndose muy deprimida. En general, las estudiantes mujeres estuvieron 
más dispuestas para hablar de este tema. Muchas de ellas reportaron que cuando sospechaban de estos problemas, les tomaba un tiempo atreverse a conversar con sus enamorados sobre ello. Antes que conversar con sus enamoradas, ellos prefirieron negar el problema, deprimirse o tomar un trago con ellas. Por lo tanto, el alcohol se convierte en una herramienta para olvidar. Sin embargo, aunque ellos son conscientes de que el alcohol eleva los sentimientos de depresión, la expectativa de alejarse de los problemas por unos momentos justifica el beber.

Los estudiantes hombres, con frecuencia, experimentaron problemas sexuales en sus relaciones. En su caso las exigencias al parecer son otras porque existe la presión por tener sexo. Los amigos generan todo un círculo de tensión sobre el tema, sobre todo entre aquellos que aún no han pasado por la experiencia.

\section{Temas financieros}

Tal y como se infirió de las discusiones de grupos focales, el dinero no apareció como un problema importante para las estudiantes mujeres quienes, en su mayoría, eran apoyadas financieramente por sus padres. En contraste, los estudiantes hombres sí experimentaron la falta de recursos económicos como un problema importante. Aunque ellos también recibían dinero de sus padres, sentían que necesitaban más dinero para cumplir con las expectativas de rol masculinas. La falta de dinero fue experimentada como una limitación para sus estudios, así como para sus interacciones sociales. Para afrontar estos problemas, los estudiantes hombres buscaron oportunidades de ganar dinero, lo que resultó en una gran preocupación por conseguir trabajo. Además, los participantes hombres de NSE medio alto reportaron que los temas financieros también afectaban las relaciones con sus padres, quienes estaban preocupados por darles dinero suficiente a sus hijos. En consecuencia, 
estos estudiantes se sentían desconsolados porque "todo gira alrededor del dinero".

\section{Dinero}

Tanto los participantes hombres como mujeres fueron conscientes de la dificultad para conseguir trabajo. Aún cuando ganar experiencia más que dinero es la razón principal para conseguir trabajo, parece difícil hacerlo sin una referencia o experiencia previa. A la mayoría de los participantes les gustaría trabajar en el campo que estudiaron, pero existe mucha aprehensión y frustración debido a la falta de oportunidades.

\section{Discriminación racial}

Las tensiones étnicas no aparecieron espontáneamente como un tema problemático y no pareció ser un tema de preocupación. Sin embargo, existieron algunos comentarios acerca de las diferencias en la ropa de las personas provenientes de diferentes etnias, así como sobre los estilos apropiados al vestir. Esto fue especialmente notorio entre las estudiantes mujeres de NSE medio, quienes se sentían presionadas para vestir a la moda, pero también sentían que era "diferente" para las chicas de diferente grupo étnico o religión. Los participantes de NSE medio alto reportaron ser discriminados por no ser blancos (e. g. no se les permitía entrar en discotecas), pero expresaron que "otros habían pasado por eso también”. Por otro lado, ellos a su vez discriminaban a otros que eran "mitad cholos, mitad blancos". 


\section{Salud}

Las estudiantes mujeres de NSE medio alto fueron las únicas que resaltaron el tema de la salud y sólo lo hicieron porque el facilitador se refirió a la pérdida extrema de peso como una enfermedad. Los desórdenes alimenticios y los problemas asociados con dietas poco balanceadas, tales como reprimir el hambre o comer poco debido a estrés psicosomático, parecieron preocupar a las chicas de este nivel socio económico en particular. Fuera de los problemas de salud relacionados con el peso, los dolores de cabeza, las reacciones en la piel, la pérdida del cabello, caspa o gastritis fueron mencionados como problemas psicosomáticos generados por la preocupación por los estudios. Los participantes hombres del NSE alto también reportaron la anorexia y las enfermedades sexuales como problemas en las mujeres.

\section{Consumo de alcohol}

Con relación al tipo de bebida consumida, la gente joven de NSE medio (tanto hombres como mujeres) usualmente bebe cerveza, ron, sangría, vodka y vino. La sangría es más popular entre las chicas y el vodka entre los chicos. Todos concordaron en que cuanto más dinero tienen, más cerveza consumen. Cuando es poco el dinero disponible, el ron es la bebida alcohólica elegida porque puede ser compartida por más gente e intoxica más rápido. Las chicas de NSE más alto eligen el vodka, el tequila y los cócteles, además de la cerveza y el vino. Los estudiantes hombres del mismo NSE reportaron beber cerveza y ron cuando no tienen dinero, ron y vodka cuando están con amigos y ron de buena calidad y vodka cuando están con chicas.

La decisión sobre qué clase de bebida alcohólica beber depende de la cantidad de dinero disponible y del grupo de amigos. 
Las bebidas alcohólicas pueden ser compradas en los supermercados o en los bares y cantinas cerca de la universidad. En general, se considera muy sencillo comprar bebidas alcohólicas. De acuerdo a los estudiantes del NSE más alto, era más difícil comprar alcohol en el pasado.

El alcohol es consumido con mayor frecuencia los jueves o viernes o en los cumpleaños, cuando terminan los exámenes o cuando hay una fiesta universitaria. Las celebraciones ocurren por lo menos una vez a la semana. Utilizar alcohol en estas ocasiones es motivado por el hecho de que beber con los amigos aumenta la interacción, mientras que si no hay alcohol, nadie habla. Beber reduce la presión de los estudios y el trabajo.

De acuerdo con los participantes de los cuatro grupos focales, el consumo de alcohol es fomentado por el grupo de pares. Los pares que beben alcohol son aquellos con los que los estudiantes salen y se divierten, y los amigos con frecuencia invitan a otros a beber. Respecto a la presión explícita de los pares, se notó que se espera que aquel que tiene algo que celebrar beba alcohol y se fuerza al resto del grupo a beber con él / ella debido a "este día especial". De esta manera, existe una influencia significativa en ambas vías. Los estudiantes hombres también indicaron que la persona que bebe más en el grupo presiona a los demás y "si tú no lo haces, eres un maricón". Sin embargo, cuando se les preguntó si sentían que debían beber aunque no quisieran, la mayoría de estudiantes hombres de NSE medio alto respondieron que no lo sentían así y que eso era una decisión personal. Por el contrario, cuando los amigos no están bebiendo, esto influye en los otros miembros del grupo y éstos no beben tampoco. 


\section{Expectativas frente al alcohol}

En los cuatro grupos, consumir alcohol fue espontáneamente asociado con diversión (e. g. bailar, compartir buenos momentos con los amigos, etc.). Otras expectativas con respecto al uso de alcohol que se mencionaron en los grupos son alivio del estrés, olvido de los problemas, sentirse relajado, perder las inhibiciones e incrementar la autoconfianza. Del análisis de contenido las siguientes categorías de expectativas pueden ser distinguidas:

\section{Entretenimiento}

Esta categoría incluye la idea de salir en grupo con los amigos para divertirse, pasar un buen rato, sentirse feliz, "ponerse pilas" y estar alegre.

Perder las inhibiciones y sentir confianza en sí mismo

Esta categoría incluye la idea de darse ánimo, darse valor para hacer algo que de otra manera no se haría tal como iniciar una conversación con alguien del sexo opuesto. Se espera que al beber alcohol, uno se sienta lo suficientemente seguro para expresar lo que uno quiere, y así facilitar la relación con la gente en general. Sin embargo, perder las inhibiciones también implica que uno puede "sobrepasar límites", es decir que uno puede ponerse muy feliz, pero también sentirse muy mal.

Alivio del estrés y olvido de los problemas

Otra razón para beber es el olvidar los problemas diarios (e. g. de la universidad, de la familia, las dificultades económicas). El 
alcohol ayuda a ver las cosas de manera diferente, a olvidar los problemas, a evitar confrontar los problemas y a relajarse.

Aceptación por parte del grupo

Para algunos participantes, la expectativa de que el grupo los aceptará mejor si beben con otros miembros de dicho grupo es un incentivo importante para beber alcohol. Sin embargo, en algunos grupos resaltó que esto depende de la personalidad de cada miembro del mismo.

Control de la cólera y/o las emociones

Algunos participantes sintieron que el alcohol los ayudaba a controlar la cólera y otras emociones. Si uno siente animadversión con respecto a alguien, uno puede "dejarla de lado" al beber alcohol.

Estas expectativas sobre el alcohol, que en su mayor parte son positivas, reflejan las dimensiones contenidas en el AEQ-A. La idea de que utilizar alcohol fomenta la diversión y eleva la aceptación de los otros refleja la expectativa del facilitación social, mientras que la idea de alivio del estrés está ligada a la expectativa de relajación y reducción de la tensión, ambas, dimensiones del AEQ-A. Otra expectativa contenida en este cuestionario, mejorar las habilidades cognitivas y motoras, se refleja en la idea de que el alcohol puede ayudar a controlar las emociones negativas como la cólera. Otras dimensiones del AEQ-A, tales como la desinhibición, la elevación de la activación o la dificultad cognitiva y motora, no aparecieron espontáneamente en las discusiones de los grupos focales. 


\section{Autoeficacia}

Aunque la autoeficacia para rechazar el alcohol es mencionada como un determinante importante en la literatura, comentarios no espontáneos fueron hechos por los participantes de los grupos focales. Para inferir sus expectativas sobre este tema, los comentarios fueron relacionados con los ítems para rechazar beber de la escala de autoeficacia. Los comentarios pueden ser resumidos como sigue:

En la mayoría de ocasiones, la autoeficacia para rechazar beber no fue vista como un problema. Por ejemplo, ante la dificultad de no beber cuando los amigos lo están haciendo se respondió con comentarios tales como: "bebo porque me gusta", "dejo de beber si no me siento bien", y "salgo del lugar y no bebo". Igualmente, la habilidad para dar buenas razones para no beber se manifestó en comentarios como: "a veces, cuando no quieres beber es porque tienes responsabilidades o problemas de salud, por lo que no te preocupa decirles eso". No estar seguro de cuándo dejar de beber provocó comentarios como: "sería un alcohólico si no pudiera hacerlo. La gente no solo bebe por presión, por ejemplo, sería una descortesía cuando estás en una reunión familiar". Junto con ello, la habilidad para dar una respuesta convincente cuando los demás se están burlando de uno por no beber no fue vista como un problema y generó comentarios como: "si no quiero beber, doy alguna excusa o simplemente digo que no". De la misma manera, estar en una fiesta o esperando en un café o bar y no beber, tampoco fue visto como una dificultad y se resolvió con respuestas tipo: "no, prefiero bailar o buscar un grupo en el que me pueda presentar. Además, la gente nunca va a las fiestas por sí misma" o "si tienes que esperar en un bar, entonces sí. Si te aburres, puedes pedir algo (un trago) mientras esperas". 
Otros aspectos de la autoeficacia sí fueron reconocidos como problemáticos. Por ejemplo, en respuesta al no resistir beber cuando se fuma, se reconoció que “...se debe al sabor de la bebida y el cigarro juntos. Es un hábito que tiene la gente: cuando beben, fuman, o viceversa". La referencia a la personalidad se hizo con relación a otros ítems de la autoeficacia. Por ejemplo, en respuesta a rechazar una bebida ofrecida, una respuesta típica fue: "tú puedes rechazar el alcohol, pero si te presionan, puedes terminar aceptando, mientras que otros no". La capacidad para resistir el deseo de beber, aún cuando se esté muy cansado, ansioso o bajo presión, generó respuestas tales como: "hay gente que bebe cuando está ansiosa. Es más fácil no beber cuando estás cansado que cuando estás ansioso" y "uno no bebe para emborracharse sino para relajarse. No se bebe por la bebida sino por la diversión; cuando uno bebe se divierte; uno se olvida un poco de todo".

En resumen, estas respuestas indican que aun cuando algunos aspectos de la autoeficacia para rehusarse a beber eran conocidos por los participantes del grupo focal, ellos generalmente no los consideraron como determinantes importantes en la decisión de beber o no.

\section{Discusión}

El propósito principal de este estudio fue explorar los hábitos de consumo de alcohol de un grupo de estudiantes universitarios en Lima e identificar los principales determinantes de su conducta de consumo, con la visión de desarrollar programas de prevención efectivos. Mediante el uso de una aproximación cualitativa que incluyó grupos focales, fue posible observar los determinantes del uso de alcohol mencionados en la literatura, tales como los problemas y estresores que experimentan los adolescentes en su vida diaria, las expectativas que tienen sobre el consumo de alcohol, la 
percepción de la presión de los pares y la autoeficacia percibida para rechazar el alcohol, tomando en consideración su contexto social y cultural. Dada la importancia de las características y procesos en el ambiente social y (sub)cultural para determinar conductas saludables, prestar atención a la sensibilidad cultural y a la especificidad de los temas podría ayudar a desarrollar programas de prevención específicos y efectivos.

Los hallazgos derivados de los grupos focales sugieren que, en sentido amplio, los temas que según la literatura favorecen o impiden el uso del alcohol entre los adolescentes, se encuentran en gran medida entre los estudiantes peruanos también, aún cuando deben tomarse en cuenta elementos culturales específicos.

Tomando en cuenta específicamente los estresores de vida, los problemas experimentados por los universitarios peruanos reflejan enormemente aquellos experimentados por los adolescentes occidentales. Al igual que sus pares europeos y americanos, los adolescentes peruanos experimentan problemas en la universidad, al prepararse para el futuro, en las relaciones con sus padres y pares, en su tiempo libre, al tratar de establecer relaciones con el sexo opuesto y con su propia forma de ser. Sin embargo, dos áreas adicionales parecen ser fuentes particulares de estrés para los estudiantes peruanos, las dificultades económicas y (para las estudiantes mujeres) lo relacionado con el control del peso. Lo primero probablemente se relaciona con la precaria situación económica en el Perú durante la última década, mientras que lo último se relaciona con la importancia que se le da a la apariencia física en la cultura latina. Por otro lado, lo relacionado con la apariencia física y el peso corporal empieza a surgir en otros países occidentales y está siendo visto como un motivador para el uso de ciertas sustancias, tales como el tabaco (Van den Broucke, Lambert \& Verduyckt, 2004). En cualquier evento, ambos estresores deberían ser tomados en cuenta cuando se considere el afrontamiento 
de los estresores de vida como un determinante del uso de alcohol entre los estudiantes universitarios peruanos.

Con respecto a las expectativas sobre el alcohol, aquellas que parecen prevalecer entre los estudiantes peruanos son: consumir alcohol provee entretenimiento y diversión, ayuda a liberar inhibiciones e incrementa la autoconfianza, alivia el estrés y las tensiones, ayuda a relajarse, facilita el contacto social y la aceptación del grupo, y ayuda a controlar la cólera y otras emociones negativas. Estas expectativas son en su mayoría positivas. En mucho, ellas reflejan las dimensiones contenidas en el Cuestionario de Expectativas sobre el Alcohol versión Adolescente (AEQ-A), ya que dos aspectos (notablemente, la expectativa de que el alcohol puede favorecer o limitar la conducta social, y que el alcohol promueve la relajación o la tensión de la reducción) reflejan directamente dimensiones contenidas en el AEQ-A, mientras que una tercera expectativa surgida de los grupos focales (aquella de que el alcohol mejora las habilidades cognitivas y motoras) es muy parecida a un tercer factor del mismo. Por lo tanto, mientras que la versión adolescente del AEQ provee de una herramienta útil para evaluar las expectativas sobre el alcohol entre los estudiantes peruanos, una total apreciación de los factores subyacentes para usar el alcohol entre ellos requiere prestar atención especial a las expectativas que parecen ser más específicas para ellos tales como el placer, la diversión, la pérdida de las inhibiciones, el incremento de la autoconfianza y el incremento de la aceptación del grupo.

Finalmente, aunque la percepción de la autoeficacia de los individuos que rehúsan beber alcohol con frecuencia es considerada un determinante importante, ello no fue confirmado por los resultados de este estudio. Primero, no surgieron comentarios espontáneos sobre este tema en los grupos focales. Segundo, aunque los participantes conocían algunos temas relacionados con la autoeficacia para rehusar beber, ellos no fueron considerados 
importantes al decidir beber o no. Esta falta de conocimiento podría atribuirse a la especificidad de los ítems que fueron utilizados para provocar las respuestas; por ejemplo, en el sentido en que las situaciones presentadas no fueron relevantes para los participantes. Debido a que la autoeficacia se refiere al control percibido o a la capacidad para comportarse como a uno le gustaría, es difícil evaluarla para una conducta que uno no está interesado en desarrollar, o que se refiere a una situación en la que uno nunca se encuentra. Por otro lado, los hallazgos de este estudio cualitativo sugieren que la autoconfianza para rechazar el alcohol no es percibida como un determinante principal del consumo del mismo por los estudiantes universitarios peruanos.

\section{Referencias}

Brown, S., Goldman, M., Inn, A. \& Anderson, L. (1980). Expectations of reinforcement from alcohol: Their domain and relation to drink patterns. Journal of Consulting and Clinical Psychology, 48, 419-426.

CEDRO. (1993). Opiniones sobre drogas en el Perú población urbana (Monografía de investigación $\mathrm{N}^{\circ}$ 8). Lima: Autor.

CEDRO. (1997). Epidemiología de drogas en la población perua$n a$ - 1995 (Encuesta en hogares). (Monografía de investigación $\left.\mathrm{N}^{\circ} 15\right)$. Lima: Autor.

Christiansen, B., Goldman, M. \& Inn, A. (1982). Development of alcohol-related expectancies in adolescents: Separating pharmacological from social-learning influences. Journal of Consulting and Clinical Psychology, 50, 3, 336-344.

Goldman, M., Brown, S. \& Christiansen, B. (1987). Expectancy theory: Thinking about drinking. En H. Blane \& E. Leonard (Eds.), Psychological theories of drinking and alcoholism (pp.181-226). Nueva York: Guilford Press. 
Johnson, V. (1988). Adolescent alcohol and marijuana use: A longitudinal assessment of a social learning perspective. American Journal on Drug and Alcohol Abuse, 14, 419-439.

Marks, D. F., Murray, M., Evans, B. \& Willig, C. (2000). Health psychology: Theory, research and practice. Londres: Sage.

Minugh, A. \& Harlow, L. (1994). Substance use clusters in a college sample: A multitheoretical approach. Journal of Substance Abuse, 6, 45-66.

Murdock, G. P. (1980). Theories of illness: A world survey. Pittsburgh, PA: University of Pittsburgh Press.

O' Conell, D. \& Patterson, H. (1989). A survey of current college alcohol abuse programs, attitudes and training needs. Journal of Alcohol and Drug Education, 43, 2, 61-69.

Oei, T., Fergusson, S. \& Lee, N. (1998). The differential role of alcohol expectancies and drinking refusal self-efficacy in problem and nonproblem drinkers. Journal of Studies on Alcohol, 59, 704-711.

Organización Mundial de la Salud. (1994). Alcohol y salud pública. Barcelona: J. R. Prous.

Petraitis, J., Flay, B. R. \& Miller, T. Q. (1995). Reviewing theories of adolescent substance use: Organizing pieces in the puzzle. Psychological Bulletin, 117, 67-86.

Saavedra, A. (1997). Comorbilidad en el alcoholismo (Monografía de investigación $N^{\circ}$ 5). Lima: Instituto Nacional de Salud Mental.

Schwarzer, R. (1993). Measurement of perceived self-efficacy. Psychometric scales for cross-cultural research. Berlin: Freie Universität Berlin.

Seiffge-Krenke, I. (1995). Stress, coping and relationships in adolescence. Mahwah, NJ: Lawrence Erlbaum.

Thorne, C. \& DeBlassie, R. (1985). Adolescent substance abuse. Adolescence, 20, 335-347.

Van den Broucke, S., Lambert, M. \& Verduyckt, P. (2004). Gender differences in the determinants of smoking among 
Consumo de alcohol y sus determinantes en estudiantes universitarios limeños

young people: A summary of the literature. Manuscrito presentado para su publicación.

Wills, T. A. \& Shiffman, S. (1985). Coping and substance use: A conceptual framework. En S. Shifman \& T. A. Wills (Eds.), Coping and substance use (pp. 3-24). Orlando, FL: Academic Press. 